\title{
Las encuestas y el género
}

\author{
Patricia Fortuny Loret de Mola
}

\section{Esperanza Tuñón Pablos, Angélica Aremy Evangelista García y Rolando Tinoco OJANGUREN (COORDS.), 2011}

Género y cultura institucional

El Colegio de la Frontera Sur, Red de Estudios de Género, México, 238 pp.

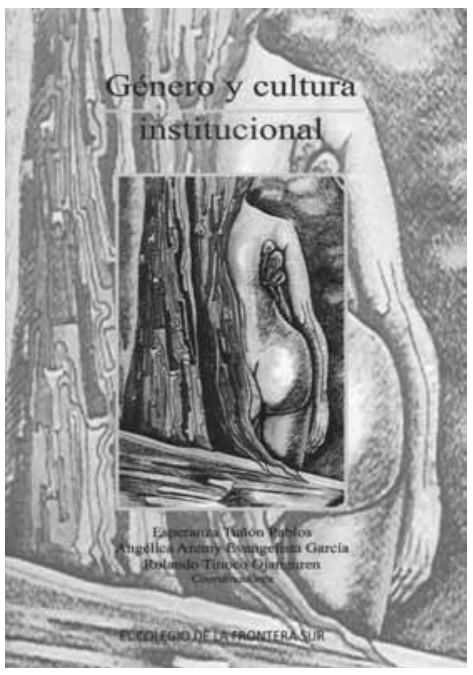

$\mathrm{I}$ nicio la reseña con la siguiente cita porque considero que se enfoca directamente en el tema tanto del libro como de la encuesta que generó el contenido de los textos que aquí reviso:

El incremento de la presencia femenina en los ámbitos laborales e institucionales no siempre resuelve los procesos culturales que sustentan las inequidades entre los sexos; en ciertos casos parecen acentuarse. [...] las

\section{Surveys and Gender}

Patricia Fortuny Loret de Mola: Centro de Investigaciones y Estudios Superiores en Antropología Social-Peninsular, Mérida, Yucatán, México mpfortuny@gmail.com

Desacatos, núm. 40, septiembre-diciembre 2012, pp. 179-183 jerarquías tradicionales ligadas al género en muchos casos se han mantenido (p. 187).

Hace algunas semanas fui parte de un examen de grado en la Universidad Nacional Autónoma de México (UNAM) y fui testigo una vez más del lenguaje sexista que está empedrado en nuestras más mexicanas y queridas instituciones. Al decidir sobre el estatus de la estudiante al final de la evaluación, el único vocablo posible era aprobado, con terminación masculina, no existía la posibilidad de escribir la forma femenina como requería el caso. Es un ejemplo más de esas ilegibles y laberínticas terminologías jurídicas que nos mantienen en el pasado y que 
se resisten al cambio. Este libro representa una etapa importante para que estos absurdos incidentes desaparezcan de las comunidades epistémicas.

Género y cultura institucional es una obra de la Colección Estudios de Género en la Frontera Sur, una iniciativa de El Colegio de la Frontera Sur. El libro tiene 238 páginas que incluyen un prefacio, siete capítulos, anexos, posfacio y la sección que nos informa sobre las y los autores. El libro en su conjunto ha sido trabajado en cada detalle con esmero, tomando en cuenta que incluye numerosas gráficas para presentar los resultados de manera ágil y eficiente. Creo que los una obra visualmente atractiva. Es una edición en la que se cuidaron todos los aspectos de la forma que, aunque no sean los únicos importantes, constituyen una parte fundamental de la cultura del libro físico. Cada capítulo, menos el introductorio, contiene decenas de gráficas que muestran la composición de la población por sexo, así como sus preferencias, aprobaciones y reprobaciones de los factores que se califican. Es un punto de partida para cualquier lector: es un trabajo fino, del cual se podrían derivar o generar otras formas de análisis.

El libro analiza los resultados obtenidos a partir de la aplicación de la Encuesta de Cultura

Institucional con Perspectiva de Género en instituciones educativas y de investigación en nuestro país en 2008. La encuesta fue responsabilidad del Instituto Nacional de las Mujeres (Inmujeres) y se aplicó en las 240 dependencias del gobierno federal. El cuestionario constaba de 96 reactivos. El Inmujeres organizó la tercera sección en ocho factores que dan testimonio de la percepción de la cultura de género en cada institución: clima laboral, comunicación inclusiva, selección de personal, salarios y prestaciones, promoción vertical y horizontal, capacitación y formación profesional, conciliación de la vida laboral, familiar y personal $y$ hostigamiento y acoso sexual. Libros como éste son una fuente primordial de consulta, no sólo para los interesados en el tema intergenérico, sino en general para conocer a la población que compone los centros educativos del país, sobre todo en el caso de las universidades estudiadas, que en orden de aparición son: la Autónoma Benito Juárez de Oaxaca, la Autónoma de Yucatán, la Juárez Autónoma de Tabasco y la Intercultural de Chiapas. El primer anexo contiene la explicación completa y precisa de los procedimientos y métodos utilizados en la elaboración y distribución del cuestionario. El segundo anexo es la encuesta misma con todos los reactivos incluidos. El breve posfacio resume y celebra el trabajo colaborativo entre las instituciones de educación superior y los centros públicos de investigación por haber logrado no sólo la aplicación de la encuesta, sino los capítulos que conforman el libro, al tiempo que señala los aspectos de la perspectiva de género en los que habrá que poner mayor atención en el presente y el futuro.

No soy una experta en el tema, pero acepté realizar esta reseña motivada por la curiosidad. Me considero una mera aficionada, puesto que he escrito capítulos de libros y artículos en los que atravieso por esos senderos en el tema de la religión y de la migración, mas no soy especialista. Sin embargo, pienso que precisamente por no ser especialista en el asunto puedo dar una opinión distinta que pueda llamar la atención de un público lector más amplio, porque creo que lo que interesa no es que nos lean sólo los especialistas, sino aquellos que saben menos del tema. También creo, en consecuencia, que es necesario esforzarnos por escribir con precisión, pero sobre todo con una suerte de convicción de que lo que hacemos debe ser inteligible para todos. En temas como éste, en el que se mezclan la discusión sobre género con las instituciones educativas, las políticas públicas y la organización, que al menos para mí resultan en ocasiones ásperos 
o difíciles de entender - aunque estén referidos en última instancia a la vida cotidiana en el trabajo- debe hacerse un doble esfuerzo por lograr una narrativa atractiva y, por qué no, conmovedora para cualquier lector.

El primer capítulo, escrito por Circe Romero Medina y Rolando Tinoco Ojanguren, contiene las reflexiones teóricas sobre la cultura organizacional y la perspectiva de género. Los autores presentan tres metodologías para observar, medir y cambiar las relaciones de género en las instituciones educativas. El capítulo es relevante en tanto que nos muestra los avances en este campo, si bien me parece que su lectura resulta una actividad muy laboriosa para las personas no familiarizadas con los términos especializados del texto. En otras palabras, los autores podrían haber transmitido más información con un lenguaje más sencillo y con ejemplos concretos que realmente desvelaran las relaciones de discriminación que ocurren en la mayoría de los espacios institucionales.

Los dos textos siguientes se ocupan de las 26 entidades -instituciones de investigación, de docencia, de formación de recursos humanos y de divulgación de la ciencia- que integran la red de centros públicos de investigación del Consejo Nacional de Ciencia y
Tecnología (Conacyt) en México. A diferencia de las universidades ubicadas generalmente en un solo estado, estos centros están distribuidos en todo el país. En el segundo capítulo, Edith Kauffer Michel analiza los resultados de la encuesta en el Centro de Investigaciones y Estudios Superiores en Antropología Social (CIESAs), en seis de sus siete sedes. Kauffer explica que una parte de la base de datos, aquella que revelaría el perfil de los entrevistados, ha quedado en manos del Inmujeres, razón por la cual hay preguntas que quedan sin respuesta, puesto que es imposible cruzar las variables sin esa parte de los datos. Lo mismo sucedió para El Colegio de la Frontera Sur (Ecosur). A pesar de este problema, la autora examina los resultados de seis de siete sedes del CIESAS con base en los ocho factores señalados y encuentra una diferenciación, que se resume así: en la sede del Distrito Federal coincide el grado de insatisfacción en hombres y mujeres; en la unidad Pacífico Sur, Oaxaca, hubo satisfacción convergente; en las unidades Occidente y Golfo - Guadalajara y Jalapa, respectivamente - se encontró satisfacción masculina e insatisfacción femenina; en las sedes Sureste y Peninsular - San Cristóbal de Las Casas y Mérida, respectivamente-, el resultado fue inverso al anterior. Esta heterogeneidad permite a la autora hablar de una geografía de la cultura institucional con especificidades locales, más que de una cultura institucional homogénea.

En el capítulo 3, Georgina Sánchez Ramírez y Angélica Aremy Evangelista García analizan los resultados en El Colegio de la Frontera Sur, que cuenta con cinco unidades: dos en el estado de Chiapas - San Cristóbal de Las Casas y Tapachula-, una en Chetumal, Quintana Roo, una en Campeche y otra en Villahermosa, Tabasco. Llama la atención que la Unidad Villahermosa del Ecosur recibió mayor número de respuestas entre los empleados. En Villahermosa contestaron la encuesta más de $88 \%$ de los trabajadores, y en la Unidad de San Cristóbal, la más antigua - la diferencia es amplia y por eso la menciono-, menos de la mitad de los que ahí laboran, es decir, $40 \%$. Desde mi punto de vista, esto es un indicador importante no sólo de una geografía institucional, como la llamó Kauffer en el capítulo anterior, sino de las especificidades locales $y$, sobre todo, del interés que históricamente ha tenido esta unidad por las cuestiones referidas al género. En Ecosur, el CIESAS y en la Universidad Autónoma de Yucatán (UADY) existe una suerte de consenso respecto de los factores "hostigamiento" y "acoso sexual", en cuanto a que hacen falta medidas institucionales precisas 
para prevenir y sancionar este tipo de problemas. Ecosur ha realizado avances en esta tarea, como otorgar una licencia de paternidad al personal académico y administrativo por diez días hábiles de descanso con salario íntegro desde 2010.

A primera vista, la Universidad Autónoma Benito Juárez de Oaxaca (UABJo) parece contar con un panorama muy distinto e incluso contrastante al de los dos centros de investigación descritos antes, en cuanto a los componentes de población femenina y masculina. Las autoras del capítulo 4, María Leticia Briseño Maas y María Guadalupe Pérez Aguilar, desglosan los resultados de la institución grande con más de 25000 estudiantes desde nivel medio superior hasta el posgrado. En cuanto a la plantilla del personal de esta institución, cabe destacar que hay muchos desequilibrios en la distribución intergenérica. Por ejemplo, en puestos de dirección de escuelas, facultades, institutos y sistemas de educación abierta y a distancia, sólo $21 \%$ están ocupados por mujeres, mientras que la proporción restante de $79 \%$ es encabezada por hombres. Cuando las mujeres cumplen el papel de "jefes" se trata de las áreas tradicionalmente femeninas, como enfermería e idiomas. La distribución de los profesores en las distintas

escuelas y facultades es igualmente desequilibrada: encontramos una mayoría aplastante de varones, con la excepción de la Escuela de Enfermería y Obstetricia. Después de presentarnos las gráficas donde aparecen los resultados de la encuesta, las autoras concluyen diciendo que "el espacio universitario no se puede comprender de forma aislada, [...] son un reflejo político, social y cultural en el que se desenvuelven" (p. 105). Las desigualdades de género están presentes y se manifiestan de manera estructural, no sólo en número, sino a través de acciones concretas.

Landy Santana Rivas y Leticia Paredes Guerrero son las autoras de "La percepción de género en la Universidad Autónoma de Yucatán”, capítulo 5, el más extenso del libro, que incluye además el mayor número de gráficas, en las que se desglosan tanto la composición de la población que labora en la institución como los resultados de la encuesta. En este caso el panorama es más alentador e igualitario que en la universidad anterior. De todas las facultades, sólo tres presentan una apabullante mayoría de varones: Medicina Veterinaria y Zootecnia, Ingeniería y Matemáticas. El resto de los departamentos, escuelas y facultades tienen un amplio número de mujeres y en muchos de éstos ellas son mayoría, aunque en números absolutos los hombres constituyan 55\% del personal de la Universidad Autónoma de Yucatán (UADY): "En cinco dependencias se guarda un equilibrio entre el número de empleados hombres y mujeres, en siete existen más mujeres que hombres y en 14 la mayoría son hombres" (p. 111, gráfica 1).

Julita Elemí Hernández

Sánchez y Renán García Falconi trabajaron sobre la cultura institucional y su relación con la perspectiva de género en la Universidad Juárez Autónoma de Tabasco (UjaT), en el capítulo 6: "En 2009 esta casa de estudios atendió a 38 mil 16 estudiantes, de quienes $58 \%$ eran mujeres y $42 \%$ hombres" (p. 160). A diferencia de los textos revisados previamente, en éste los autores comienzan con una crítica al Plan de Desarrollo Estratégico 2008-2012. Dicen en forma abierta que dicho Plan no exhibe ninguna respuesta o acción concreta con perspectiva de género y sugieren que los estatutos de la institución sean revisados y actualizados. Existe una distribución de 40/60 y las mujeres son la población menor. En este capítulo encontramos una radiografía de la muestra que respondió a la encuesta: 102 hombres y 128 mujeres. Las conclusiones de este capítulo podrían ser leídas como radicales, aunque también pueden ser muy 
realistas. Cito una frase que resume la posición:

Las mujeres mexicanas trabajan fuera de casa, pero como pudimos ver con los resultados de este estudio, [...] no deben violar su supuesta naturaleza de fragilidad, no deben tener deseos de poder y jamás olvidar su "sublime obligación" de ser madre y esposa fiel (p. 185).

El capítulo 7 se titula "Vivencias de la inequidad. Estudio de caso en la Universidad Intercultural de Chiapas" y fue escrito por Susie Morales Moreno y Minerva Yoimi Castañeda Seijas, citadas al principio de esta reseña. La Universidad Intercultural de Chiapas (UNICH) fue creada en 2005 a la par de otras de la misma naturaleza en el país. El cuestionario fue respondido por un universo de 113 personas, casi por el mismo número de personas por cada sexo. Después de ofrecernos la sociodemografía de quienes atendieron la encuesta, las autoras pasan a las respectivas calificaciones que recibieron los ocho factores por sector, hombres y mujeres. En contraste con las demás universidades pero también con los centros públicos de investigación, esta institución obtuvo una calificación de baja a media en la evaluación de su cultura institucional. Puede notarse que en todos los factores las calificaciones otorgadas por las mujeres fueron menores que las de los varones. En el reactivo sobre el respeto a la opinión de hombres y mujeres en la institución, que forma parte del factor percepción del clima laboral según sexo, la distancia es de casi 20 puntos, 69 la población femenina y 89 la masculina. En casi todos los factores se observan importantes diferencias entre las respuestas de ellas y las de ellos, tal vez con la excepción del factor "promoción vertical u horizontal". Como afirman las autoras, existe un visible alejamiento entre lo que se vive y se percibe y los lineamientos generales de la política institucional.

Todos los capítulos, sin importar los resultados que tuvieron en la encuesta, sugieren que este diagnóstico debiera servir para implementar medidas y sanciones concretas para alcanzar una verdadera relación igualitaria entre los y las trabajadoras de estas instituciones. Los datos y las gráficas deben servir para que autoridades y empleados lleguen a consensos sobre cómo mejorar el clima laboral y la percepción intergenérica. El libro nos da una idea muy cercana de lo que sucede en nuestros centros académicos y de investigación. De ahí que la difusión de estos resultados sea una tarea imprescindible. También pienso que el diagnóstico realizado todavía nos ofrece una parte de la realidad no acabada del todo, la siguiente tarea debe ser - ahora sí- utilizar una metodología con una orientación más cualitativa que nos permita ver más de cerca los verdaderos problemas sobre el clima laboral. Las entrevistas, la etnografía y otras formas cualitativas de recopilación de información serían capaces de poner al descubierto los conflictos y negociaciones que se gestan entre los sexos donde yace el poder, la resistencia y la subordinación, mismos que los métodos de encuesta son incapaces de mostrar en toda su magnitud. 